\title{
«Hoster la contraincte de retoricque»: notes de lecture sur le "Nouveau Répertoire de mises en prose (XIV $-\mathrm{XVI}^{\mathrm{e}}$ siècle)"
}

\section{Michelle Szkilnik}

\section{(2) OpenEdition}

\section{Journals}

\section{Édition électronique}

URL : http://journals.openedition.org/studifrancesi/4272

DOI : 10.4000/studifrancesi.4272

ISSN : 2427-5856

\section{Éditeur}

Rosenberg \& Sellier

\section{Édition imprimée}

Date de publication : 1 septembre 2016

Pagination : 273-277

ISSN : 0039-2944

\section{Référence électronique}

Michelle Szkilnik, « «Hoster la contraincte de retoricque»: notes de lecture sur le "Nouveau Répertoire de mises en prose (xIV -xvI e siècle)" », Studi Francesi [En ligne], 179 (LX | II) | 2016, mis en ligne le 01 septembre 2017, consulté le 18 septembre 2020. URL : http://journals.openedition.org/studifrancesi/ 4272 ; DOI : https://doi.org/10.4000/studifrancesi.4272

\section{(c) (i) (9)}

Studi Francesi è distribuita con Licenza Creative Commons Attribuzione - Non commerciale - Non opere derivate 4.0 Internazionale. 


\title{
DISCUSSIONI E COMUNICAZIONI
}

\section{«Hoster la contraincte de retoricque»: notes de lecture sur le "Nouveau Répertoire de mises en prose $\left(X I V^{e}-X V T^{e} \text { siècle }\right)^{9 ! 1}$}

\begin{abstract}
The thorough updating and reshaping of Doutrepont's Répertoire de mises en prose recently published by Maria Colombo Timelli, Barbara Ferrari, Anne Schoysman and François Suard, is the starting point for this brief consideration on the process of prosification at the end of the Middle Ages. Perusing the prologues edited in the new Répertoire shows that authors whose verse works were turned into prose had usually fallen into oblivion (even Chrétien de Troyes). Fifteenth-century remanieurs argue that to be rescued, earlier texts need to be brought up to date: their language modernized and their form changed to suit the tastes of a new audience. Modern readers, so they say, like prose better than verse. Verse is obscure and prolix, while prose is elegant and unconstrained by rhetoric.
\end{abstract}

Le vaste mouvement de mise en prose qui s'amorce au XIII ${ }^{e}$ siècle pour s'étendre à quasiment tous les textes narratifs, comme le répertoire réalisé sous la direction de Maria Colombo Timelli, Barbara Ferrari, Anne Schoysman et François Suard le montre si bien, pose une première question: pourquoi est-il si systématique en France? On ne trouve rien de tel dans les pays voisins, ni en Angleterre, ni en Allemagne, ni en Italie, par exemple. Certes on y pratique la prose, mais il n'y a pas de transfert du vers à la prose à l'échelle de ce qui se passe en langue française. Cette question n'a pas de réponse évidente, et ce n'est pas ici le lieu d'en exposer tous les aspects'. Il convient toutefois de signaler d'emblée la spécificité de la prose française dans son impérialisme face au vers. Elle est, après tout, à l'origine du travail originel de Georges Doutrepont ${ }^{3}$ et de l'établissement du Nouveau Répertoire. Le phénomène de mise en prose, comme le signale l'avant-propos ${ }^{4}$, a pour conséquence d'effacer les différences génériques qui pouvaient encore subsister par exemple entre chansons de geste et romans. À bien des égards, il n'y avait plus guère de différences thématiques entre chansons et romans depuis le XIII siècle. Est-ce la conscience de cette communauté de motifs, voire de personnages qui a conduit à harmoniser les formes ou qui du moins a facilité cette harmonisation?

Un second point intéressant, que je signalerai sans m'y attarder, est que la poussée de la prose n'aboutit pas d'emblée à la disparition du vers narratif. L'invention du «dit» au XIV ${ }^{e}$ siècle le montre bien. Le dit utilise le vers de manière beaucoup plus complexe que les romans arthuriens des XII et XIII siècles, le Roman d'Alexandre,

(1) Nouveau Répertoire de mises en prose (XIV XVI siècle), sous la direction de M. Colombo TImelli, B. Ferrari, A. Schoysman et F. Suard, Paris, Classiques Garnier, 2014. Titre abrégé désormais en NRMP.

(2) Elle a fait l'objet du colloque que Catherine Croizy-Naquet et moi avons organisé au mois de mars 2015: «Rencontres du vers et de la prose: conscience poétique et mise en texte», dont les actes seront publiés chez Brepols.

(3) G. Doutrepont, Les mises en prose des épopées et des romans chevaleresques du XIV au XVI siècle, Bruxelles, Palais des Académies (impr. de G. Thone), 1939 [Genève, Slatkine Reprints, 1969 et 2011].

(4) NRMP, p. 9. 
voire la chanson de geste, puisqu'il joue des formes, introduisant des pièces lyriques complexes, voire fait alterner vers et prose. Le dit va donc redonner une certaine vigueur à l'octosyllabe. Mais le développement de cette forme nouvelle, vite arrêtée au demeurant, n'empêche pas la progression écrasante de la prose au XV siècle. Le dit est pris en charge par un «je» à la forte présences. On peut avancer l'hypothèse que les auteurs qui ont illustré cette forme, Guillaume de Machaut, Froissart, Christine de Pizan, ont des personnalités telles qu'elles rendent quasi impossible la mise en prose de leurs œuvres. Tout au plus peut-on les détourner partiellement comme le roman de Cardenois s'inspire du Jugement du roi de Behaigne.

Cette remarque m'amène à un troisième point que je développerai davantage: le passage à la prose est sans aucun doute favorisé par l'effacement du nom ou du renom des auteurs originaux. Le cas de Chrétien de Troyes est de ce point de vue exemplaire. Les deux mises en prose effectuées au milieu du Xv' siècle à la cour de Bourgogne, celle d'Erec et Enide et celle de Cligès, ne mentionnent aucun nom d'auteur ${ }^{7}$. Le Perceval de 1530 ne connaît pas Chrétien non plus ${ }^{8}$. L'auteur de cette grosse compilation, qui reprend, outre le Conte du Graal, la version longue de la Première Continuation, la Continuation de Wauchier de Denain, celle de Manessier, l'Elucidation et le Bliocadran, évoque bien le travail de Chrétien en reprenant du reste assez fidèlement le prologue du Conte du Graal, mais il omet le nom du Champenois qu'il appelle «aucun docte orateur» et «chroniqueur» alors même qu'il conserve celui du commanditaire, Philippe de Flandres'. Il sait que l'entreprise de Chrétien a été poursuivie puisqu'il reprend l'hypothèse de la mort de Chrétien et de Philippe de Flandres avant l'achèvement du projet et avance que la comtesse Jeanne de Flandres a souhaité poursuivre le travail de son aïeul en confiant cette tâche «à ung sien familier orateur nommé Mennessier». Non seulement le nom de Chrétien n'est pas donné, alors même que le prologue du Conte l'indique, mais son rôle est réduit à celui d'un traducteur-compilateur rédigeant et mettant par écrit un ouvrage que lui aurait donné le comte de Flandres, tandis que Manessier est investi à son tour de la même fonction par la comtesse Jeanne. On sait que le prologue du Conte mentionne en effet le don d'un ouvrage. Le metteur en prose du $\mathrm{XVI}^{\mathrm{e}}$ siècle ne s'interroge pas sur l'existence ni la nature de la source primitive dont il accepte comme une évidence la réalité. En somme, pour lui, Chrétien et Manessier n'ont rien fait d'autre que ce que lui réalise quelques siècles plus tard «en ensuyvant au plus prés selon ma possibilité et pouair le sens de mes predecesseurs translateurs, comme ay trouvé par leur escript». Il s'inscrit sans hésitation dans la lignée des deux auteurs devenus comme lui de simples «translateurs» et dont il s'approprie les propos: «Car, comme ay dict, ledict Philippes conte de Flandres estoit tant rempli de charité que riens ne voioit où elle deust estre gardee...» (je souligne). Ce n'est évidemment pas lui qui le premier a mentionné la charité de Philippe ni développé la parabole du semeur, mais bien Chrétien de Troyes.

L'attitude de ce compilateur est typique de celle de la plupart des «metteurs en prose ${ }^{10}$ qui manifestent une ignorance totale, et peut-être même un désintérêt tout aussi entier, à l'égard de l'identité des auteurs qu'ils dériment. Certes, un grand

(5) Voir la définition que Jacqueline CerquiligniToulet donne de ce genre dans Grundriss der Romanischen Literaturen des Mittelalters, VIII/1, sous la direction de D. PoIRION, Heidelberg, Carl Winter Universitätsverlag, 1988, pp. 86-94.

(6) NRMP, notice pp. 131-134.

(7) NRMP, notices pp. 215-222 et pp. 183-189.

(8) NRMP, notice pp. 681-688.

(9) Le prologue est édité p. 683 du NRMP.

Toutes mes citations sont empruntées à cette page.
(10) Prosateurs, dérimeurs, remanieurs, translateurs, «metteurs en prose»: il est difficile de savoir comment désigner ceux qui se sont livrés aux opérations de mise en prose, précisément parce que cellesci sont de nature très variée. Sur ce point, voir A. Combes, «L'emprise du vers dans les mises en prose romanesques (XIII-XV ${ }^{e}$ siècle)», Le Moyen Âge par le Moyen Âge, même. Réception, relectures et réécritures des textes médiévaux dans la littérature française des $X I V^{\circ}$ et XV siècles, Paris, Champion, 2012, pp. 115-140. 
nombre des œuvres dérimées sont anonymes. C'est le cas par exemple de toutes les chansons de geste. Mais dans les quelques cas où il y a un auteur connu, son nom n'apparaît qu'exceptionnellement. Jean Wauquelin par exemple ne cite ni Alexandre de Paris ni Lambert le Tors. À plusieurs reprises, il précise du reste qu'il ignore le nom de l'auteur originel dont il transpose l'œuvre. Pour sa mise en prose d'Alexandre, il indique ainsi que sa source est un «livre tout rimet dont [il] ne sça[it] le nom de l'auteur» ${ }^{11}$. Il s'excuse de même manière dans sa mise en prose de Gérard de Roussillon $^{12}$. Le prosateur de Florent et Octavien allègue de son côté que sa source est «un livre mis en ryme dont [il] ne sça[it] le nom de l'auteur» ${ }^{13}$. Le dérimeur de la chanson de Bertrand Du Guesclin ne mentionne pas Cuvelier bien que son dérimage soit quasi contemporain de la version originale en vers ${ }^{14}$. L'auteur du Cardenois utilise Machaut sans le nommer, alors que le souvenir du poète devait être encore bien vivant. L'auteur de la Prose 2 du Roman de Troie reprend assez fidèlement le prologue de Benoît de Sainte-Maure et rappelle la transmission du texte en citant Homère et le légendaire Cornélius, mais il omet le passage où Benoît se nomme ${ }^{15}$.

J'ai examiné tous les prologues édités dans le Répertoire pour voir s'il y avait quelques auteurs épargnés par cet implacable effacement. La moisson est maigre. Le nom de Guillaume de Digulville est conservé par Jean Galopes ainsi que dans la mise en prose du clerc anonyme d'Angers ${ }^{16}$. Sans surprise, Jean Molinet évoque Guillaume de Lorris et Jean de Meun (mais pas dans son prologue) ${ }^{17}$. L'auteur d'une des proses du Florimont dit dérimer le livre «qu'Aymez de Varennes fist de grejoys en françoys» ${ }^{18}$. Plus souvent quand des noms (ou des titres, ce qui est plus fréquent) apparaissent dans les prologues, ils jouent le rôle d'autorités qui masquent la véritable source du prosateur. Des ouvrages en latin sont ainsi mentionnés. Wauquelin cite Jacques de Guise et ses Annales qu'il utilise très partiellement dans sa mise en prose d'Alexandre ${ }^{19}$. Vincent de Beauvais et son Miroir historial figurent dans plusieurs prologues ${ }^{20}$. Comme je l'ai déjà signalé, les conditions de transmission des textes expliquent sans doute en grande partie l'effacement des auteurs originels. Toutefois l'exemple du Perceval de 1530 et du Roman de Troie Prose 2 montrent aussi que pour beaucoup de prosateurs les œuvres en vers qu'ils ont recueillies constituent d'abord un réservoir d'histoires et d'exemples que la transmission manuscrite au fil des siècles a frappés d'anonymat et qu'ils ont donc toute liberté de reprendre et d'adapter aux goûts nouveaux de leur public.

Comment les «translateurs» rendent-ils compte de l'opération de mise en prose dont le répertoire montre combien elle est variée, du véritable dérimage à la refonte intégrale? Là encore les prologues offrent des remarques diverses. Certains prosateurs prétendent qu'ils traduisent du latin, du provençal, du picard, de l'espagnol ${ }^{21}$. Les expressions «mettre en langage maternel» ou en «prose familiere» reviennent à plusieurs reprises ${ }^{22}$. Ce que disent ces expressions ou la demi-vérité de la traduction d'une autre langue, c'est que le français des $\mathrm{XII}^{\mathrm{e}}$ et $\mathrm{XIII}^{\mathrm{e}}$ siècles est bel

(11) NRMP, p. 22. Notice pp. 15-31.

(12) NRMP, p. 340 . Notice pp. 332-245

(13) NRMP, p. 250. Notice pp. 245-252.

(14) NRMP, notice pp. 99-113.

(15) NRMP, p. 800. Notice pp. 797-803.

(16) NRMP, notices pp. 669-680 et 643-667.

(17) NRMP, notice pp. 761-772.

(18) NRMP, p. 271. Notice pp. 266-284

(19) NRMP, p. 25.

(20) Comme celui de l'Alexandre de Jean Wauquelin (NRMP, p. 25), ou celui du Fierabras de Jean Bagnyon (NRMP, p. 232).

(21) Du latin: Galien le Restoré (NRMP, p. 288),
Florimont (NRMP, p. 262), Gilles de Chin (NRMP, p. 412), Jean d'Avesnes (NRMP, p. 513), le Myreur des histors de Jean d'Outremeuse (NRMP, p. 598); du wallon: Gérard d'Euphrate de Jean Maugin (NRMP , p. 385), de l'espagnol: Giglan de Claude Platin (NRMP, p. 404); du «viel picart»: Jourdain de Blaves (prose 2, NRMP, p. 529); du provençal: Gérart de Nevers (NRMP, p. 324).

(22) NRMP, p. 250, 340, 395. Les prosateurs parlent de leur langage vulgaire (NRMP, p. 310), du bon vulgaire français (NRMP, pp. 737), de leur «prose familiere» (NRMP, p. 683).

(23) NRMP, p. 232. 
et bien une langue étrangère pour les prosateurs du $X v^{e}$ et pour leurs lecteurs. Jean Bagnyon le déclare explicitement qui décrit sa source comme «ung roman ancien en rime, aulcunement en lengaige estrange ${ }^{23}$. Revient fréquemment l'idée que le texte ancien est obscur. Dans le prologue de sa Geste du chevalier au cygne, Berthault de Villebresme évoque l'«ancienne rime et assez obscur lengaige difficille a compregnoir et entendre» ${ }^{24}$. L'auteur du Renaut de Montauban bourguignon explique que son livre a été «reduict de vieil langaige corrumpu en bon vulgaire françoys» ${ }^{25}$. Dans de très nombreux cas, le prosateur explique qu'il veut rendre le texte accessible à son public et que l'opération de translation n'a pas d'autre objet.

Il n'est pas toujours facile de déterminer si c'est l'emploi du vers ou l'état de langue qui rend le texte ancien incompréhensible au public moderne. Dans quelques cas toutefois, le prosateur oppose clairement vers et prose. Sans surprise, l'argument du vers menteur a quasiment disparu au $X^{\mathrm{e}}$ siècle. On le rencontre encore à la fin du $\mathrm{XIII}^{\mathrm{e}}$ dans la première mise en prose du Roman de Troie «je la (cette histoire) translaterai en françois et non pas par rime ne par vers ou il covient par fine force avoir maintes menchoignes com font ces menestriez qui de lor lengues font maintefois rois et amis solacier» ${ }^{26}$. La prose bourguignonne du Renaut de Montauban semble pourtant lier vers et mensonges quand l'auteur du prologue déclare vouloir composer son histoire en prose, après avoir étudié «croniques, vielles gestes, livres anciens rimés» et assure qu'il n'est ni «gengleur ne controuveur de menchonges» ${ }^{27}$. Mais la condamnation du vers est plus largement contenue dans le rejet des anciennes gestes.

L'un des reproches plus fréquemment adressés au vers à la fin du Moyen Âge, c'est sa «prolixité»: le vers est inutilement bavard. Jean Wauquelin dans le prologue de la Belle Hélène de Constantinople explique qu'il va «retrenschier et sincoper les prolongacions et motz inutiles qui souvent sont mis et boutez en telles rimes» ${ }^{28}$. Les mots inutiles sont-ils les chevilles? Le prologue de la mise en prose de la Chanson de Bertrand du Guesclin allègue lui aussi une brièveté nécessaire et parle d'«abregier le langage et evader prolixité» ${ }^{29}$. Jean Galopes et le prosateur de Godefroy de Bouillon disent de même. Le cas de ce dernier texte est très intéressant car le prosateur reconnaît le charme du vers mais il a choisi la prose par souci d'abrègement: «l'ai commenchie sans rime pour l'estoire avoir plus abregie et si me sanle que le rime est mout plaisans et mout bele mais mout est longue» ${ }^{30}$. Il est rare que les metteurs en prose rendent ainsi explicitement hommage au vers, même si certains d'entre eux mêlent des vers à la prose. Par ailleurs, il est amusant de voir les écrivains avancer le souci de brièveté, alors que la prose amplifie souvent considérablement les textes en vers. Mais la brièveté est moins une question de quantité que d'efficacité et, de ce point de vue, la prose semble aller plus directement au but que le vers ${ }^{31}$.

Le second argument justifiant le choix de la prose est le plaisir des lecteurs. Les lecteurs «modernes» aiment mieux la prose que le vers. À lire les prologues, il semblerait que les commanditaires ont pesé sur le choix de la forme. Même si Jean Wauquelin et David Aubert par exemple ne le disent pas explicitement, ils mentionnent

(24) NRMP, p. 395. Notice pp. 393-399.

(25) NRMP, p. 737. Notice pp. 717-746.

(26) NRMP, p. 787. Notice pp. 773-795.

(27) NRMP, p. 727.

(28) NRMP, p. 56. Notice pp. 51-60.

(29) NRMP, p. 93.

(30) NRMP, p. 420. Notice pp. 419-425.

(31) Sur l'esthétique de la brièveté que les écrivains médiévaux semblent chérir, voir Faire court. L'Esthétique de la brièveté dans la littérature du
Moyen Âge, sous la direction de C. CROIZY-NAQuet, L. Harf-Lancner et M. Szkilnik, Paris, Presses de l'Université de la Sorbonne Nouvelle, 2011.

(32) NRMP, pp. 56 et 482.

(33) NRMP, p. 240.

(34) NRMP, p. 456. Notice pp. 453-474.

(35) NRMP, p. 862.

(36) NRMP, p. 255. Notice pp. 253-258.

(37) NRMP, p. 154 . Notice pp. 151-164.

(38) NRMP, p. 477. Notice pp. 474-493. 
le plus souvent un «livret rimet» à eux remis par leur patron et qu'ils vont tourner en prose, soulignant au passage leur volonté de se plier aux désirs de celui-ci. Le dérimage est sans doute l'une des exigences du mécène ${ }^{32}$. Certains prosateurs sont plus précis. Le prologue de Florent et Lyon déclare ainsi clairement que «plusieurs gens preignent plus grant plaisir a lyre romans en prose que en rime» ${ }^{33}$. De même, selon le prologue du Guillaume d'Orange en prose: «plus est le laingage plaisant prosé que rimé» ${ }^{34}$. Le prologue du Théséus de Cologne avance un argument semblable ${ }^{35}$ tandis que le prosateur de Florient et Florette allègue son propre plaisir: «la matiere du present livret m'est plus agreable a lire en prose que en rime» ${ }^{36}$.

Pourquoi les lecteurs préfèrent-ils la prose au vers? Parce qu'elle est plus élégante. Dans les Chroniques et conquêtes de Charlemagne, David Aubert vante la qualité des textes en prose: «aucuns font nouvelles transcriptions et mutation de rime en prose, en termes si bien couchies qu'il semble que rethorique propre leur mette ou cuer ce qu'ilz escripvent» ${ }^{37}$. Dans la version anonyme de l'Histoire de Charles Martel copiée par David Aubert, il est dit que «princes et autres seigneurs appettent plus la prose que la ryme, pour le langaige quy est plus entier et n'est mie tant contraint $\gg^{38}$. On voit apparaittre ici une explication du goût nouveau pour la prose: elle est plus claire, plus libre, moins «contrainte». Cet aveu permet d'affirmer que ce n'est pas seulement l'ancienneté de la langue, mais bien aussi la forme vers qui rebuterait le lecteur moderne. Jean Wauquelin ne dit pas autre chose quand il prétend «hoster la contraincte de retoricque» afin d'embellir l'histoire ${ }^{39}$. Philippe de Vigneulles lie la prose et l'abrègement qu'elle permet à un progrès de l'esprit humain: «weullent les gens de maintenant avoir chose abregee et plaisante, car les esperit deviegne tout les jours plus agus et soubtille $($ sic $) \gg^{40}$. On trouve une déclaration voisine chez Jean Molinet qui souligne la qualité de la nouvelle langue: «nostre langaige est fort agensy, fort mignon et renouvelés ${ }^{41}$.

Ainsi pour les prosateurs et leurs lecteurs, la prose est libre et moderne, le vers démodé et empesé par une vieille rhétorique. Déclaration étonnante à une époque où s'épanouissent les grands rhétoriqueurs, dont Jean Molinet fait partie! Et l'on sait que Philippe de Vigneulles n'hésite pas lui non plus à recourir au vers. Il faut sans doute comprendre que ce n'est pas le vers qui est démodé, mais le récit en vers, comme le dit explicitement le prologue de Florent et Lyon pour qui il y a «plus grant plaisir a lyre romans en prose que en rime» (c'est moi qui souligne ${ }^{42}$.

Après la tentative sans grand lendemain du dit narratif, les formes vers et prose se spécialisent d'une manière qui ne nous surprendra pas: à la prose le discours savant et scientifique, les traités militaires, les compilations historiques et les œuvres narratives. Au vers, l'expression du moi, le lyrisme, l'intime. Cette répartition ne s'est pas imposée d'emblée et au XIV ${ }^{\mathrm{e}}$ siècle par exemple elle n'est certainement pas aussi tranchée que ma formulation le laisse entendre. Pour le XV siècle et peut-être surtout pour le XVI ${ }^{\mathrm{e}}$, on pourrait également introduire des nuances. N'empêche: la pression de la prose est inexorable et les metteurs en prose dont le Répertoire présente le travail, ont à la fois accompagné et accéléré le mouvement qui a assuré le triomphe de la prose.

On ne peut qu'être reconnaissant à l'équipe qui a repris, mis à jour, complété, amélioré le travail pionnier de Georges Doutrepont. Le Nouveau Répertoire est un outil précis qui permet d'accéder rapidement à un ensemble d'informations essentielles, de comparer les mises en prose et de mener des enquêtes fructueuses sur des textes encore mal connus faute d'éditions modernes. 